\title{
Lacunary Statistical Convergence of Order alpha and Lacunary Strongly Summable Sequences of Order alpha of Generalized Difference Sequences
}

\author{
Mikail Et $t^{1,{ }^{*}}$,Hacer Şengül ${ }^{2}$, and Muhammed Çınar ${ }^{3}$ \\ ${ }^{1}$ Department of Mathematics, Firat University, Elazı̆̆, Turkey \\ ${ }^{2}$ Faculty of Education, Harran University, Osmanbey Campus, Şanlıurfa, Turkey \\ ${ }^{3}$ Department of Mathematic Education, Muş Alparslan University, Muş, Turkey
}

\begin{abstract}
The notion of the $\alpha$ th order $\Delta_{i}^{m}$ - lacunary statistical convergence and $\alpha$ th order lacunary strongly $\left(\Delta_{i}^{m}, p\right)$ - summable sequences was introduced by Altınok et al. [1]. They also gave significant inclusion correlation associated with the aforementioned sequence spces. In this paper, our aim is to exploit some other important relations between the given notions.
\end{abstract}

\section{Introduction}

The idea of statistical convergence was firstly determined by Fast [12] and redefined independently by Schoenberg [28]. The concept of the density of the set of positive integers $\mathbb{N}$ was used as the main motivation to create this idea. For any subset $F$ of the $\mathbb{N}$, the density of $F$ is defined as

$$
\beta(F)=\lim _{n \rightarrow \infty} \frac{1}{n} \sum_{l=1}^{n} \omega_{F}(l)
$$

where $\omega_{F}$ is the characteristic function of $F$. It is needless to say that this definition is valid only for the existence of the limit. Let $y=\left(y_{l}\right)$ be a given any sequence then it is statistically converged to $R$, if for every $\varepsilon>0, \beta\left\{l \in N:\left|y_{l}-R\right| \geq \varepsilon\right\}=0$. Furthermore, the relation and some application of this definition with the summabilitiy theory was presented by using the perspective of the sequence space by Çinar et al. ([3], [6], [30]), Connor [5], Fridy [14], Et et al. ([2], [11], [30]), Işık et al. ([17], [18], [19], [20], [21])). Gadjiev and Orhan [16] stated firstly the order of the sequence's statistical

* Corresponding author: mikailet68@gmail.com 
convergence. Based on this research, Çolak [4] studied the $\alpha$ th order of the statistical convergence and $\alpha$ th order of the strong $p$-Cesàro summability.

The lacunary sequence is defined by $h_{k}=\left(l_{k}-l_{k-1}\right) \rightarrow \infty$ as $k \rightarrow \infty$, where $\gamma=\left(l_{k}\right)$ is an increasing sequence of integer. The interval denoted by $\gamma$ is determined by $I_{k}=\left(l_{k-1}, l_{k}\right]$ and $q_{k}$ is the abbreviation of the ratio of $\frac{l_{k}}{l_{k-1}}$ ([13], [15], [20], [29]). The introduction of the difference sequence space and its modification and extension were recently conducted by Kızmaz [23] and Et and Çolak [7]. Tripathy et al. [31] obtained the generalized shape of the difference sequences by considering the former difference sequences in the following form

$$
Y\left(\Delta_{i}^{m}\right)=\left\{y=\left(y_{l}\right):\left(\Delta_{i}^{m} y_{l}\right) \in Y\right\}
$$

where $Y$ is regarded as a sequence, $\mathrm{i}$ and $\mathrm{m}$ are any two arbitrary positive integers, and $\Delta_{i}^{m} y_{l}=\sum_{w=0}^{m}(-1)^{w}\left(\begin{array}{c}m \\ w\end{array}\right) y_{l+i w}$.

We rather choose to take $Y\left(\Delta^{m}\right)$ in lieu of $Y\left(\Delta_{i}^{m}\right)$ and $\Delta^{m} y$ in lieu of $\Delta_{i}^{m} y$ if we assume that $i=1$. Other well known studies can be found in the following papers ([8], [9], [10], [17], [22], [25]).

\section{Main Results}

This section is the core of the study since it contains main results ans theorems.

Definition 2.1. [1] Let $\gamma=\left(l_{k}\right)$ be a lacunary sequence such that $m$ and $i$ are choosen as arbitrarily non-negative integers and $0<\alpha \leq 1$ be given. If a real number $R$ is defined as follows

$$
\lim _{k \rightarrow \infty} \frac{1}{h_{k}^{\alpha}}\left|\left\{l \in I_{k}:\left|\Delta_{i}^{m} y_{l}-R\right| \geq \varepsilon\right\}\right|=0
$$

then the sequence $y=\left(y_{l}\right)$ is said to be $\Delta_{i}^{m}-$ lacunary statistically convergent of order $\alpha$ (or $S_{\gamma}^{\alpha}\left(\Delta_{i}^{m}\right)-$ convergent) .

Thus it is obvious that $S_{\gamma}^{\alpha}\left(\Delta_{i}^{m}\right)-\lim y_{l}=R$. The set of all $\Delta_{i}^{m}-$ lacunary statistically convergent sequences will be denoted by $S_{\gamma}^{\alpha}\left(\Delta_{i}^{m}\right)$.

$\Delta_{i}^{m}$ - lacunary statistical convergence is well defined for $0<\alpha \leq 1$, but it is not well defined for $\alpha>1$ in general. It is evidently true that every $\Delta^{m}-$ convergent sequence is lacunary $\Delta_{i}^{m}-$ statistically convergent of order $\alpha \quad(0<\alpha \leq 1)$, but the converse does not hold.

Definition 2.2. [1] Let $\gamma=\left(l_{k}\right)$ be a lacunary sequence such that $m$ and $i$ are choosen as arbitrarily non-negative integers and $\alpha, p>0$ be given. If a real number $R$ is defined as follows 


$$
\lim _{k \rightarrow \infty} \frac{1}{h_{k}^{\alpha}} \sum_{l \in I_{k}}\left|\Delta_{i}^{m} y_{l}-R\right|^{p}=0
$$

then a sequence $y=\left(y_{l}\right)$ is said to be strongly $w_{\gamma}^{\alpha}\left(\Delta_{i}^{m}, p\right)$ - summable (or lacunary strongly $\left(\Delta_{i}^{m}, p\right)$-summable of order $\left.\alpha\right)$.

Thus it is obvious that $w_{\gamma}^{\alpha}\left(\Delta_{i}^{m}, p\right)-\lim y_{l}=R$. The set of all strongly $w_{\gamma}^{\alpha}\left(\Delta_{i}^{m}, p\right)-$ summable sequences of order $\alpha$ will be denoted by $w_{\gamma}^{\alpha}\left(\Delta_{i}^{m}, p\right)$. If we take $\gamma=\left(2^{k}\right)$ then we write $w^{\alpha}\left(\Delta_{i}^{m}, p\right)$ instead of $w_{\gamma}^{\alpha}\left(\Delta_{i}^{m}, p\right)$.

For the following theorems we omit to give detailed proof since it is easily proved by using standard methods.

Theorem 2.3. Let $0<\alpha \leq 1$ and $\gamma=\left(l_{k}\right)$ be a lacunary sequence. If $\limsup \operatorname{su}_{k} \frac{l_{k}}{l_{k-1}^{\alpha}}<\infty$, then $w_{\gamma}^{\alpha}\left(\Delta_{i}^{m}, p\right) \subset w^{\alpha}\left(\Delta_{i}^{m}, p\right)$.

Theorem 2.4. Let $\gamma=\left(l_{k}\right)$ and $\gamma^{\prime}=\left(s_{k}\right)$ be two lacunary sequences such that $I_{k} \subset J_{k}$ for all $k \in \mathbb{N}$ and let $\alpha$ and $c$ be fixed real numbers such that $0<\alpha \leq c \leq 1$,

(i) If

$\liminf _{k \rightarrow \infty} \frac{g_{k}^{\alpha}}{\ell_{k}^{c}}>0$

then $S_{\gamma}^{\beta},\left(\Delta_{i}^{m}\right) \subseteq S_{\gamma}^{\alpha}\left(\Delta_{i}^{m}\right)$,

(ii) If

$\lim _{k \rightarrow \infty} \frac{\ell_{k}}{g_{k}^{c}}=1$

then $S_{\gamma}^{\alpha}\left(\Delta_{i}^{m}\right) \subseteq S_{\gamma}^{c},\left(\Delta_{i}^{m}\right)$, where $I_{k}=\left(l_{k-1}, l_{k}\right], \quad J_{k}=\left(s_{k-1}, S_{k}\right], \quad g_{k}=l_{k}-l_{k-1}$ and $\ell_{k}=s_{k}-s_{k-1}$.

Theorem 2.5. Let $\gamma=\left(l_{k}\right)$ and $\gamma^{\prime}=\left(s_{k}\right)$ be two lacunary sequences such that $I_{k} \subseteq B_{k}$ for all $k \in \mathbb{N}, \alpha$ and $c$ be fixed real numbers such that $0<\alpha \leq c \leq 1$ and $0<p<\infty$. Then we get

(i) If (1) holds then $w_{\gamma}^{\beta},\left(\Delta_{i}^{m}, p\right) \subset w_{\gamma}^{\alpha}\left(\Delta_{i}^{m}, p\right)$,

(ii) If (2) holds and $y \in \ell_{\infty}\left(\Delta_{i}^{m}\right)$ then $w_{\gamma}^{\alpha}\left(\Delta_{i}^{m}, p\right) \subset w_{\gamma}^{\beta},\left(\Delta_{i}^{m}, p\right)$.

Theorem 2.6. Let $\gamma=\left(l_{k}\right)$ and $\gamma^{\prime}=\left(s_{k}\right)$ be two lacunary sequences such that $I_{k} \subseteq B_{k}$ for all $k \in \mathbb{N}, \alpha$ and $c$ be fixed real numbers such that $0<\alpha \leq c \leq 1$ and $0<p<\infty$. Then 
(i) Let $(1)$ holds, if a sequence is strongly $w_{\gamma}^{\beta},\left(\Delta_{i}^{m}, p\right)-$ summable to $R$, then it is $S_{\gamma}^{\alpha}\left(\Delta_{i}^{m}\right)$ - convergent to $R$,

(ii) Let (2) holds, if a $\Delta_{i}^{m}$ - bounded sequence is $S_{\gamma}^{\alpha}\left(\Delta_{i}^{m}\right)$ - convergent to $R$ then it is strongly $w_{\gamma}^{\beta},\left(\Delta_{i}^{m}, p\right)$ - summable to $R$.

Theorem 2.7. If $y \in w^{\alpha}\left(\Delta_{i}^{m}, p\right) \cap w_{\gamma}^{\alpha}\left(\Delta_{i}^{m}, p\right)$ and $\limsup \frac{l_{k}}{l_{k-1}^{\alpha}}<\infty$, then $w_{\gamma}^{\alpha}\left(\Delta_{i}^{m}, p\right)-\lim y_{l}=w^{\alpha}\left(\Delta_{i}^{m}, p\right)-\lim y_{l}=R$.

Proof. Let $w_{\gamma}^{\alpha}\left(\Delta_{i}^{m}, p\right)-\lim y_{l}=R$ and $w^{\alpha}\left(\Delta_{i}^{m}, p\right)-\lim y_{l}=R^{\prime}$, and suppose that $R \neq R^{\prime}$. Since $\limsup \frac{l_{k}}{l_{k-1}^{\alpha}}<\infty$, from Theorem 2.3 we already know that $w_{\gamma}^{\alpha}\left(\Delta_{i}^{m}, p\right) \subset w^{\alpha}\left(\Delta_{i}^{m}, p\right)$. Since $\left(\Delta_{i}^{m} y-R^{\prime}\right) \in w_{\gamma, 0}^{\alpha}\left(\Delta_{i}^{m}, p\right)$, it follows that $\left(\Delta_{i}^{m} y-R^{\prime}\right) \in w_{0}^{\alpha}\left(\Delta_{i}^{m}, p\right)$ and therefore $\frac{1}{t^{\alpha}} \sum_{b=1}^{t}\left|\Delta_{i}^{m} y_{b}-R^{\prime}\right| \rightarrow 0$. Then we have $\frac{1}{t^{\alpha}} \sum_{b=1}^{t}\left|\Delta_{i}^{m} y_{b}-R^{\prime}\right|+\frac{1}{t^{\alpha}} \sum_{b=1}^{t}\left|\Delta_{i}^{m} y_{b}-R\right| \geq \frac{1}{t^{\alpha}}\left|R-R^{\prime}\right|>0$,

and hence $R=R$.

\section{Results Related to Modulus Function}

Nakano developed the mathematical background of the notion of the modulus [26]. Then this notion is used for the construction of some sequence spaces by Maddox [24] and Ruckle [27]. Now, we present the inclusion relations among the sets of lacunary strongly $\left(\Delta_{i}^{m}, p\right)$-summable sequences of order $\alpha$ and $\Delta_{i}^{m}$ - lacunary statistically convergent sequences of order $\alpha$ with respect to the modulus function $f$.

Definition 3.1. Let $f$ be a modulus function, $p=\left(p_{l}\right)$ be a sequence of strictly positive real numbers and $\alpha>0$. Now we define

$w_{\gamma}^{\alpha}\left(\Delta_{i}^{m},(p), f\right)=\left\{y=\left(y_{l}\right): \lim _{k \rightarrow \infty} \frac{1}{g_{k}^{\alpha}} \sum_{l \in I_{k}}\left[f\left(\left|\Delta_{i}^{m} y_{l}-R\right|\right)\right]^{p_{l}}=0\right.$, for some $\left.R\right\}$.

If $y \in w_{\gamma}^{\alpha}\left(\Delta_{i}^{m},(p), f\right)$, then we say that $y$ is strongly $w_{\gamma}^{\alpha}\left(\Delta_{i}^{m},(p), f\right)$-summable with respect to the modulus function $f$. If $p_{l}=p$ for all $l \in \mathbb{N}$ and $f(y)=y$ we shall write $w_{\gamma}^{\alpha}\left(\Delta_{i}^{m}, p\right)$ instead of $w_{\gamma}^{\alpha}\left(\Delta_{i}^{m},(p), f\right)$ and in the special case $f(y)=y$ we shall write $w_{\gamma}^{\alpha}\left(\Delta_{i}^{m},(p)\right)$ instead of $w_{\gamma}^{\alpha}\left(\Delta_{i}^{m},(p), f\right)$. 
In the following theorems we shall assume that the sequence $\left(p_{l}\right)=p$ is bounded and $0<g=\inf _{l} p_{l} \leq p_{l} \leq \sup _{l} p_{l}=G<\infty$.

Theorem 3.2. Let $m$ and $i$ be two non-negative integers, $\gamma=\left(l_{k}\right)$ be a lacunary sequence, $\alpha$ and $c$ be fixed real numbers such that $0<\alpha \leq c \leq 1$ and $f$ be a modulus function, then $w_{\gamma}^{\alpha}\left(\Delta_{i}^{m},(p), f\right) \subset S_{\gamma}^{c}\left(\Delta_{i}^{m}\right)$.

Proof. Let $y \in w_{\gamma}^{\alpha}\left(\Delta_{i}^{m},(p), f\right)$ and let $\varepsilon>0$ be given and $\Sigma_{1}$ and $\Sigma_{2}$ denote the sums over $l \in I_{k},\left|\Delta_{i}^{m} y_{l}-R\right| \geq \varepsilon$ and $l \in I_{k},\left|\Delta_{i}^{m} y_{l}-R\right|<\varepsilon$, respectively. Since $g_{k}^{\alpha} \leq g_{k}^{c}$ for each $k$ we may write

$$
\begin{aligned}
\frac{1}{g_{k}^{\alpha}} \sum_{l \in I_{k}}\left[f\left(\left|\Delta_{i}^{m} y_{l}-R\right|\right)\right]^{p_{l}} & =\frac{1}{g_{k}^{\alpha}}\left[\sum_{1}\left[f\left(\left|\Delta_{i}^{m} y_{l}-R\right|\right)\right]^{p_{l}}+\sum_{2}\left[f\left(\left|\Delta_{i}^{m} y_{l}-R\right|\right)\right]^{p_{l}}\right] \\
& \geq \frac{1}{g_{k}^{c}}\left[\sum_{1}\left[f\left(\left|\Delta_{i}^{m} y_{l}-R\right|\right)\right]^{p_{l}}+\sum_{2}\left[f\left(\left|\Delta_{i}^{m} y_{l}-R\right|\right)\right]^{p_{l}}\right] \\
& \geq \frac{1}{g_{k}^{c}}\left|\left\{l \in I_{k}:\left|\Delta_{i}^{m} y_{l}-R\right| \geq \varepsilon\right\}\right| \min \left([f(\varepsilon)]^{g},[f(\varepsilon)]^{G}\right) .
\end{aligned}
$$

Hence $y \in S_{\gamma}^{c}\left(\Delta_{i}^{m}\right)$.

Theorem 3.3. Let $\gamma=\left(l_{k}\right)$ be a lacunary sequence, $m$ and $i$ be two non-negative integers and $0<\alpha \leq 1$. If $\lim _{k \rightarrow \infty} \frac{g_{k}}{g_{k}^{\alpha}}=1$ and the modulus $f$ is bounded, then $S_{\gamma}^{\alpha}\left(\Delta_{i}^{m}\right) \subset w_{\gamma}^{\alpha}\left(\Delta_{i}^{m},(p), f\right)$.

Proof. Let $y \in S_{\gamma}^{\alpha}\left(\Delta_{i}^{m}\right)$ and suppose that $f$ is bounded and $\varepsilon>0$ be given. Since $f$ is bounded there exists an integer $K$ such that $f(y) \leq K$, for all $y \geq 0$. Then we may write

$$
\begin{aligned}
\frac{1}{g_{k}^{\alpha}} \sum_{l \in I_{k}}\left[f\left(\left|\Delta_{i}^{m} y_{l}-R\right|\right)\right]^{p_{l}}= & \frac{1}{g_{k}^{\alpha}}\left(\sum_{1}\left[f\left(\left|\Delta_{i}^{m} y_{l}-R\right|\right)\right]^{p_{l}}+\sum_{2}\left[f\left(\left|\Delta_{i}^{m} y_{l}-R\right|\right)\right]^{p_{l}}\right) \\
\leq & \max \left(K^{g}, K^{G}\right) \frac{1}{g_{k}^{\alpha}}\left|\left\{l \in I_{n}:\left|\Delta_{i}^{m} y_{l}-R\right| \geq \varepsilon\right\}\right| \\
& +\frac{g_{k}}{g_{k}^{\alpha}} \max \left(f(\varepsilon)^{g}, f(\varepsilon)^{G}\right) .
\end{aligned}
$$

and so $w_{\gamma}^{\alpha}\left(\Delta_{i}^{m},(p), f\right)$. 
Theorem 3.4. Let $\gamma=\left(l_{k}\right)$ be a lacunary sequence, $m$ and $i$ be two non-negative integers and $0<\alpha \leq 1$. If $y=\left(y_{l}\right)$ is strongly $w_{\gamma}^{\alpha}\left(\Delta_{i}^{m},(p), f\right)-$ summable to $R$ with respect to the modulus function $f$ and $\lim p_{l}>0$, then $w_{\gamma}^{\alpha}\left(\Delta_{i}^{m},(p), f\right)-\lim y_{l}=R$ unique.

Proof. Omitted.

Theorem 3.5. Let $\gamma=\left(l_{k}\right)$ be a lacunary sequence, $m$ and $i$ be two non-negative integers and $0<\alpha \leq 1$. The sequence spaces $w_{\gamma}^{\alpha}\left(\Delta_{i}^{m}, p\right), S_{\gamma}^{\alpha}\left(\Delta_{i}^{m}\right)$ and $w_{\gamma}^{\alpha}\left(\Delta_{i}^{m},(p), f\right)$ are neither solid nor symmetric, nor sequence algebras for $m \geq 1$.

Proof. Let $\gamma=\left(2^{k}\right)$ and $i=1$, then $\left(y_{l}\right)=\left(l^{m-1}\right) \in w_{\gamma}^{\alpha}\left(\Delta_{i}^{m}, p\right)$ but $\left(\alpha_{l} y_{l}\right) \notin w_{\gamma}^{\alpha}\left(\Delta_{i}^{m}, p\right)$ when $\alpha_{l}=(-1)^{l}$ for all $l \in \mathbb{N}$. Hence $w_{\gamma}^{\alpha}\left(\Delta_{i}^{m}, p\right)$ is not solid. The other cases can be proved on considering similar examples.

\section{References}

1. H. Altınok, M. Et, M. Işık, AIP Conference Proceedings 1926, 020004 (2018); https://doi.org/10.1063/1.5020453

2. H. Altınok, M. Et, R. Çolak, Iran. J. Fuzzy Syst. 11(5), 39-46, (2014)

3. M. Çınar, M. Karakaş, M. Et, Fixed Point Theory Appl. 2013:33, 11 pp (2013)

4. R. Çolak, Modern Methods in Analysis and Its Applications, New Delhi, India : Anamaya Pub, 2010, 121-129 (2010)

5. J.S. Connor, Analysis 8, 47-63 (1988)

6. M. Et, M. Çınar, M. Karakaş, J. Inequal. Appl., 2013:204, 8 pp (2013)

7. M. Et, R. Colak, Soochow J. Math. 21(4), 377-386 (1995)

8. M. Et, H. Altınok, Y. Altın, Appl. Math. Comput. 154(1), 167-173 (2004)

9. M. Et, A. Alotaibi, S.A. Mohiuddine, The Scientific World Journal, 535419 (2014)

10. M. Et, M. Mursaleen, M. Işık, Filomat 27(5), 789-796 (2013)

11. M. Et, B.C. Tripathy, A.J. Dutta, Kuwait J. Sci. 41(3), 17-30 (2014)

12. H. Fast, Colloq. Math. 2, 241-244 (1951)

13. A.R. Freedman, J.J. Sember M. Raphael, Proc. Lond. Math. Soc. 37(3), 508-520 (1978)

14. J.A. Fridy, Analysis, 5, 301-313 (1985)

15. J.A. Fridy, C. Orhan, Pacific J. Math. 160, 43-51 (1993)

16. A.D. Gadjiev, C. Orhan, Rocky Mountain J. Math. 32(1), 129-138 (2002)

17. M. Işık, J. Inequal. Appl. 2010, Art. ID 457892, 7 pp (2010)

18. M. Iş1k, K.E. Akbaş, J. Inequal. Spec. Funct. 8(4), 57-64 (2017)

19. M. Işıı, Math. Slovaca 61(5), 779-788 (2011)

20. M. Iş1k, K.E. Et, AIP Conference Proceedings 1676, 020045 (2015); doi: http://dx.doi.org/10.1063/1.4930471.

21. M. Işık, K.E. Akbaş, ITM Web of Conferences 13, 01024 (2017); doi: 10.1051/itmconf/20171301024

22. M. Karakaş, M. Et, V. Karakaya, Acta Math. Sci. Ser. B (Engl. Ed.) 33(6), 17111720 (2013) 
23. H. Kizmaz, Canad. Math. Bull. 24(2), 169-176 (1981)

24. I.J. Maddox, Math. Proc. Camb. Philos. Soc. 100, 161-166 (1986)

25. M. Mursaleen, R. Çolak, M. Et, J. Inequal. Appl. 2007, Art. ID 86757, 6 pp (2007)

26. H. Nakano, Proc. Japan Acad. 27, 508-512 (1951)

27. W.H. Ruckle, Canad. J. Math. 25, 973-978 (1973)

28. I.J. Schoenberg, Amer. Math. Monthly 66, 361-375 (1959)

29. H. Şengül, M. Et, Acta Math. Sci. Ser. B Engl. Ed. 34(2), 473-482 (2014)

30. H.M. Srivastava, M. Et, Filomat 31(6), 1573-1582 (2017)

31. B.C. Tripathy, A. Esi, B.K. Tripathy, Soochow J. Math. 31(3), 333-340 (2005) 